\title{
Vortex nucleation in Bose-Einstein condensates due to effective magnetic fields
}

\author{
D. R. Murray, ${ }^{1}$ P. Öhberg, ${ }^{2}$ D. Gomila, ${ }^{3}$ and S. M. Barnett ${ }^{1}$ \\ ${ }^{1}$ Department of Physics, SUPA, University of Strathclyde, Glasgow G4 ONG, United Kingdom \\ ${ }^{2}$ Department of Physics, SUPA, Heriot-Watt University, Edinburgh EH14 4AS, United Kingdom \\ ${ }^{3}$ IFISC (CSIC-UIB), Instituto de Física Interdisciplinar y Sistemas Complejos, \\ Campus Universitat Illes Balears, E-07122 Palma de Mallorca, Spain
}

(Received 11 May 2009; published 10 June 2009)

\begin{abstract}
We investigate the rotational properties of a Bose-Einstein condensate (BEC) in an effective magnetic field. The corresponding gauge potential is optically generated, and based on the adiabatic motion of the atoms. We demonstrate that the nucleation of vortices is seeded by instabilities in surface excitations and show that this picture also holds when the applied effective magnetic field is not homogeneous. The eventual configuration of vortices in the cloud depends on the geometry of the applied field.
\end{abstract}

DOI: 10.1103/PhysRevA.79.063618

PACS number(s): 03.75.Kk, 42.50.Gy

\section{INTRODUCTION}

A spectacular property of superfluid systems is their ability to support quantized vortices [1]. These can appear as flux lines in superconductors to which a sufficiently strong magnetic field has been applied. Alternatively, in the case of neutral superfluids subject to sufficiently fast external rotation, they exist as lines of vanishing condensate density around which the velocity field flow is quantized $[2,3]$. These scenarios are closely linked because the equations describing a rotating superfluid, when studied in the rotating frame, mimic those of a charged superfluid (a superconductor) in a magnetic field, with the Coriolis force playing the role of the Lorentz force.

The dilute gas BEC is an extremely useful tool for probing the underlying physics of superfluid phenomena because, in experiments, its parameters are typically much easier to manipulate than for other condensed-matter systems. Moreover, because of its diluteness, it is considerably more amenable to a theoretical treatment which accurately reflects the experimental reality $[3,4]$. A good example is in the studies of condensates rotated by an anisotropic potential, where the critical velocity for vortex nucleation has been found to coincide with dynamical instabilities in the surface mode excitations $[2,5]$. At the same time, the prospect of simulating effects related to charged particles in magnetic fields means that the rotating $\mathrm{BEC}$ continues to receive much attention [6-8].

Rotating a condensate only provides access to a limited class of problems, however, for which the effective magnetic field is spatially homogeneous in the plane perpendicular to the rotation axis. Stirring a Bose-Einstein condensate using asymmetric traps or laser "spoons" [9-11] has become a standard technique to induce vortices in condensates. The controlled stirring of a condensate can be a rather demanding task if a small number of particles are used, which is often the case for strongly correlated systems such as atomic Tonks-Girardeau [12-14] and Quantum Hall gases [6-8,15-17]. Recent proposals to create effective magnetic fields in a more direct way open the door for more wideranging studies into the interaction of degenerate quantum gases with effective magnetic fields. One method involves using lasers to alter the state-dependent tunneling amplitudes of atoms in an optical lattice to simulate an effective magnetic flux [18-20]. Another, considered here, exploits the interaction of $\Lambda$-type three-level atoms with two laser beams possessing relative orbital angular momentum in an electronically induced transparency (EIT) configuration. The corresponding vector potential shows up in the effective equation of motion [21-23] for the atoms, which sit in a nondegenerate eigenstate of the laser-atom interaction. An advantage of this method is that the vector potential, and consequently the effective magnetic field, can be shaped and controlled by appropriate modifications of the phase and intensity of the incident light [24].

In this paper we study, by numerical simulations, the influence of both homogeneous and inhomogeneous effective magnetic fields on the dynamics of a harmonically trapped Bose-Einstein condensate and observe vortex nucleation for critical parameter values. The exact dynamics are specific to the geometry of the trapping potential and effective magnetic field, but the existence of unstable modes in the spectrum of elementary excitations as a precursor to vortex nucleation is a universal feature for all cases considered.

\section{ORIGIN OF THE GAUGE POTENTIAL}

In a series of recent papers (see [22] and references therein) it has been shown how neutral atoms can experience an optically induced effective magnetic field. The underlying mechanism is geometric in its nature, and is based on the adiabatic approximation. The atomic state we are concerned with is an eigenstate of the interaction Hamiltonian and is a linear superposition of the two lowest states in a $\Lambda$ configuration, so-called dark state. If the atoms remain in this state while moving in space, i.e., adiabaticity is preserved, then the corresponding equation of motion for the atom's center of mass will acquire a vector potential [25]. For this we need two lasers, one driving each "leg" of the $\Lambda$ system. For all this to hold, any transition between the dark state and the corresponding bright state [22] needs to be suppressed. If the incident laser fields are tuned to the one- and two-photon resonances then any motion of the atoms will introduce a doppler detuning. As long as this doppler detuning is small 
compared to the total Rabi frequency, the dark state will remain stable [22]. Shape oscillations or rotations in BoseEinstein condensates typically involve velocities less than the speed of sound which is of the order of a few microns per millisecond. Hence the doppler detuning would be small compared to a typical total Rabi frequency. The lifetime of the dark state, and ultimately the gauge potential, can therefore be of the order of a few seconds [22]. Interestingly, the effective gauge potential depends only on the ratio between the Rabi frequencies and the gradient of their phase difference [22]. This results in a remarkable degree of freedom, and is indeed the key to be able to construct spatially nonuniform effective magnetic fields in charge neutral quantum gases.

One way to achieve a nonzero phase gradient in the ratio of the control and probe beam coupling strengths is to use light with orbital angular momentum. Such light beams with nontrivial intensity distribution and steep phase gradients, can be readily created by interference techniques [26] or by using spatial light modulators. Two-dimensional optical structures are in this respect straightforward to create because only a transversal section of the light beam at the position of the ultracold gas is relevant as far as the effective magnetic field is concerned.

A Laguerre-Gaussian beam, for example, has transverse field $u_{p \ell}(r, \phi) \propto r^{|\ell|} L_{p}^{|\ell|}\left(r^{2}\right) \exp \left(-r^{2}\right) \exp (i \ell \phi)$, where $L_{p}^{|\ell|}$ is the associated Laguerre polynomial, such that each photon carries angular momentum $\ell \hbar$ around the propagation axis [27], which we take to be the $z$ direction. If one or both beams are of this type and incident at a normal to the cloud of atoms trapped in the $x-y$ plane, then the induced vector potential acting on the atoms is well approximated by [28]

$$
\mathbf{A}=-\frac{\hbar \ell}{R} \alpha_{0}\left(\frac{r}{R}\right)^{\nu} e_{\phi}
$$

which corresponds to an effective magnetic field

$$
\mathbf{B}=-\frac{\hbar \alpha_{0} \ell(\nu+1)}{R^{2}}\left(\frac{r}{R}\right)^{\nu-1} e_{z} .
$$

Here $\alpha_{0}$ is the ratio of intensities of the two beams at a characteristic radius $R$. In deriving Eq. (1), we assume $\alpha_{0}$ to be small $(\$ 0.1)$ which ensures one state is much more heavily populated.

With the inclusion of the vector potential [Eq. (1)], which satisfies the condition $\boldsymbol{\nabla} \cdot \mathbf{A}=0$, the effective equation governing the atom dynamics is given by [22]

$$
i \hbar \frac{\partial \Psi}{\partial t}=\left(-\frac{\hbar^{2}}{2 M} \nabla^{2}+\tilde{V}+g|\Psi|^{2}+\frac{i \hbar}{M} \mathbf{A} \cdot \boldsymbol{\nabla}\right) \Psi
$$

where $\tilde{V}(r)=V+\frac{|A|^{2}}{2 M}$ and $g=4 \pi \hbar^{2} a / M a_{z}$ is the scaled strength of the two-body collisions between atoms and $a_{z}$ represents the thickness of the cloud in the $z$ direction. When $\nu=1$ in Eq. (1), an equation of identical form to Eq. (3) can be obtained by considering a BEC subject to a time-dependent trapping potential which is rotating at angular velocity $\Omega$, and studying the resultant Gross-Pitaevskii equation in the rotating frame in which the trapping potential is independent of time. This would correspond to an effective magnetic field which is homogeneous in the $z$ direction $\mathbf{B}=2 M \Omega e_{z}$.

\section{VORTEX NUCLEATION AND DYNAMICS OF THE QUANTUM GAS}

The possibility of studying inhomogenous effective magnetic fields $(\nu \neq 1)$ raises many interesting questions. Do we still observe vortex nucleation above a critical field strength? Does the same mechanism for vortex nucleation-attributed to instabilities of the surface mode excitations-still hold for effective magnetic fields which are inhomogeneous? If so what does the lattice configuration look like?

We shall address these questions in turn, using results of numerical simulations of the real-time dynamics of the condensate as determined by Eq. (3), where the vector potential is of the form given by Eq. (1). We shall consider the trapping potential as it appears in Eq. (3) to be of the form

$$
\tilde{V}=\frac{1}{2} M \omega_{\perp}^{2} r^{2}+\frac{\epsilon R^{2}}{\left|\cos \left(\frac{j}{4} \phi\right)\right|+\left|\sin \left(\frac{j}{4} \phi\right)\right|},
$$

that is, harmonic plus a small perturbation which is anisotropic, where $j$ gives the degree of rotational symmetry, $\phi$ is the polar angle, and $\epsilon$ is a dimensionless parameter. Potentials of this form could in principle be created using multiple beam patterns of the desired symmetry (see e.g., [26]). A harmonic potential can be achieved by a judicious choice of the external trapping potential to counteract the additional scalar potentials arising due to the light-atom interaction. Our motivation for considering potentials which are predominantly harmonic form is twofold. First, this is of the same form as the rotating-frame potential of atoms in a rotating harmonic trap, allowing for a clear comparison. Second, because we are primarily interested in vortex nucleation, it is instructive to eliminate the scalar potential which can preclude vortex nucleation in inhomogeneous magnetic fields by preventing the necessary surface mode instabilities from occurring [28].

The addition of the asymmetry in the potential distorts the flow $\mathbf{J}=\frac{\hbar}{2 i}\left(\psi^{*} \boldsymbol{\nabla} \psi-\psi \nabla \psi^{*}\right)-\mathbf{A}|\psi|^{2}$, leading to shape oscillations. In the presence of the effective magnetic field [Eq. (2)], potentials having rotational symmetry $j$ naturally couple to surface modes of multipolarity $m=j$. Beyond a critical value of $\alpha_{0} \ell$, these modes have negative energy, signifying an energetic instability. In Fig. 1 we plot the location of the surface mode energetic instabilities for three different effective magnetic fields. These instabilities were calculated in Ref. [28], for a BEC in an isotropic harmonic trap. The addition of the anisotropic term in Eq. (4) will shift the mode frequencies but not by a significant amount provided $\epsilon$ is sufficiently small.

In order to simulate experimental imperfections and to break the unrealistic levels of symmetry [29], we add small fluctuations $(\sim 0.01 \%\langle\tilde{V}\rangle \ll$ grid spacing) to the trap coordinates periodically (not more often than every $25 / \omega_{\perp}$ time units). This does not change the dynamics qualitatively but speeds up the symmetry breaking, which would otherwise occur due to growth of numerical noise [29]. In addition, the 


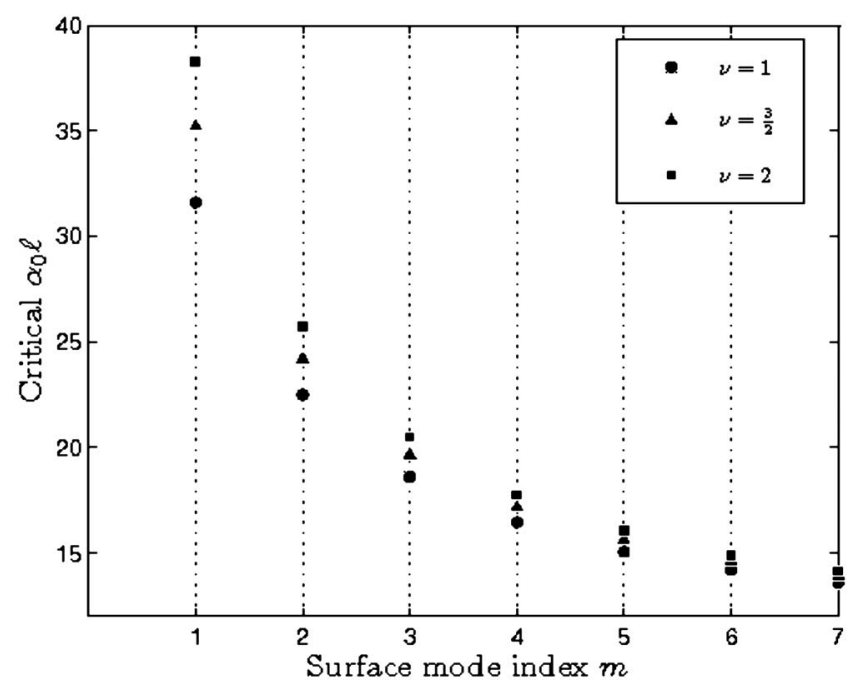

FIG. 1. Location of the energetic instability of the surface modes $m$ for three different vector potentials of the form $\mathbf{A} \sim r^{\nu} \mathrm{e}_{\phi}$. We assume here and for the remainder of the paper the parameter values $R=\left(2 \mu / m \omega^{2}\right)^{\frac{1}{2}}=1, g / \mu=1$ and $(\hbar \omega / 2 \mu)^{2}=0.001$ ( $\mu$ the chemical potential), which describe a condensate in the ThomasFermi regime. Above the critical field strength $\alpha_{0} \ell$, the mode excitation becomes negative, so that in the presence of dissipation the system may lower its energy by going into an "anomolous" mode, which can become dynamically unstable.

initial state for Eq. (3) is assumed to be real valued. The gauge potential during the initial-state calculation (using imaginary time propagation) has therefore no effect. Only during the real-time propagation will the gauge potential couple to the quantum gas and its dynamics through the weak symmetry-breaking potential in $\widetilde{V}$ and the induced fluctuations. In doing so we avoid any large amplitude oscillations in the trapped gas when introducing the gauge potential (see also Refs. [5,30,31]. for discussions on adiabatic introduction of rotations). The optically induced gauge potential does however also allow a sudden switch on of the effective magnetic fields. The gauge potential is proportional to the intensity ratio of the two incident laser beams. This makes it possible in an experiment to adjust the strength of the effective magnetic field both in time and space simply by changing the intensity of one of the laser beams.

An already well-studied problem in respect of its equivalence to rotating condensate experiments is the homogeneous effective magnetic field $(\nu=1)$ applied to a condensate in a harmonic trap with a small elliptic perturbation $[2,5,32,33]$, corresponding to $j=2$ in Eqs. (1)-(3). The trap couples to the quadrupole oscillations carrying $m=2$ units of angular momentum, which become unstable at $\alpha_{0} \ell \approx 23$ (see Fig. 1) when the cyclotron frequency associated with the effective magnetic field matches the bare quadrupole frequency in absence of the field. This induces large amplitude oscillations which are dynamically unstable, eventually leading to nucleation of vortices in the bulk of the condensate.

It is natural to contemplate whether a similar situation should occur for inhomogenous effective magnetic fields, that is, do we observe vortex nucleation due to coupling of the elliptic trap to a surface mode which becomes unstable.
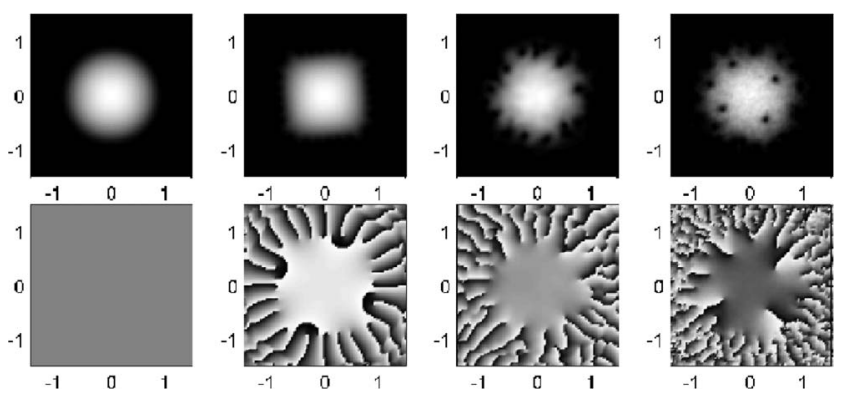

FIG. 2. Snapshots of the density (top) and phase (bottom) for an inhomogeneous effective magnetic field with $\nu=\frac{3}{2}, \alpha_{0} \ell=18.2$, $j=4$, where the time $\omega_{\perp} t=0,20,360,700$ increasing from left to right. The $m=4$ octopole surface mode is resonantly excited, and vortex nucleation is enabled by a dynamical instability. Our simulations use an easily implemented leap-frog method with $150 \times 150$ points and $(x, y) \in[-2.25,2.25]$ in units of $R$. The time step $d t$ is $0.00005 / \omega_{\perp}$.

The cases we consider are when $\nu=\frac{3}{2}$ and $\nu=2$, which both correspond to an effective magnetic field which increases with radius $r$ [see Eq. (2)]. For $\nu=2$ the $m=2$ surface mode becomes energetically unstable at $\alpha_{0} \ell \simeq 26$ (see Fig. 1). Simulations run with this vector potential indicate again a strong shape deformation after the effective magnetic field is switched on but rather than fragmentation followed by vortex nucleation and eventual crystallization of the vortex lattice, we observe a more dramatic shedding of density from the cloud. The reason for these explosive dynamics can be understood by classical arguments: the effective scalar potential "seen" by the rotating condensate has an additional quartic contribution $-\frac{|\mathbf{A}|^{2}}{2 M}$, and reaches a maximum at radius $r_{\max }$. Whenever the chemical potential $\mu$ exceeds the maximum potential energy of the trap $V\left(r_{\max }\right)$ many atoms are expelled from the cloud. In such cases the system no longer becomes manageable for either numerical studies or actual experiments. The same applies for the $\nu=\frac{3}{2}$ case, and indeed all potentials with $\nu>1$ due to the effect the additional counter potential which is steeper than harmonic.

We can however observe vortex nucleation in inhomogeneous effective magnetic fields provided we choose the potential in Eq. (4) to couple to modes higher than $m=2$. In Fig. 2, we demonstrate nucleation of vortices for the $\nu=\frac{3}{2}$ field with an asymmetric perturbation to the harmonic trap of rotational symmetry $j=4$, where $\alpha_{0} \ell$ is chosen to be slightly above the critical value as determined by Fig. 1 .

The presence of energetic instability is a necessary, but insufficient criterion for vortex nucleation; it reflects only that the wave function is no longer a local minimum of the energy above a critical $\alpha_{0} \ell$, where spontaneous creation of elementary excitations is favorable. The nucleation of vortices is due to the dynamical instability, the signature of which is a complex excitation energy, allowing small amplitude perturbations to grow exponentially in time causing a significant change to the initial state. Consistent with this picture, our studies indicate the existence of an upper as well as lower bound of $\alpha_{0} \ell$ between which vortex nucleation occurs, corresponding to a window of dynamical instability. However, the location of an energetic instability is useful for pre- 

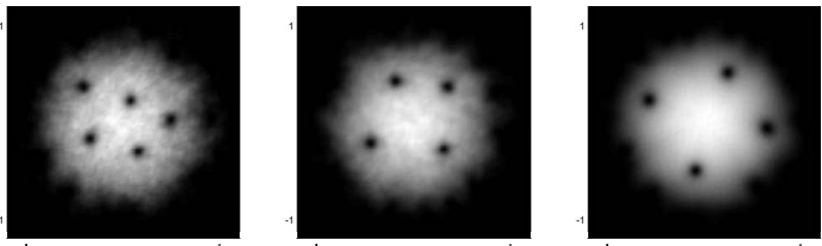

FIG. 3. Density at $t=450$ for BEC in an asymmetric trap subject to the effective magnetic field with (a) $\nu=1$ (homogeneous), (b) $\nu=\frac{3}{2}$, and (c) $\nu=2$. The parameters used in (a)-(c) were $\epsilon=0.1$, $j=7, \alpha_{0} \ell=15.5$, and $\left(\hbar \omega_{\perp} / 2 \mu\right)^{2}=0.001$. The nucleation of vortices is driven by resonant excitation of the $m=7$ mode, for which the critical $\alpha_{0} \ell$ is just below 15 for each of magnetic fields considered here (see Fig. 1).

dicting vortex nucleation because dynamical instabilities only exist in conditions of energetic instability [34].

In accord with the studies of Refs. $[33,35,36]$, we note that the width of the unstable region increases with $\epsilon$, the degree of anisotropy in the trapping potential of Eq. (4). For the parameters $\nu=2$ and $j=5$, we observe vortex nucleation within the range $\alpha_{0} \ell=15.25$ to 16.25 for $\epsilon=0.05$ and $\alpha_{0} \ell=15.0$ to 17.0 for $\epsilon=0.1$.

\section{VORTEX LATTICE}

Let us also briefly mention variation in the structure of the vortex lattice as a function of the geometry of the effective magnetic field. As is well known, the condensate in a homogenous effective magnetic field with a large number of vortices favors an Abrikosov-type lattice [37]. Even with our small number of vortices, it is clear that the lattice approaches a regular homogenous structure for $\nu=1$. In contrast to the homogeneous case, we note that for inhomogeneous effective magnetic fields with $\nu>1$ the vortices tend to be concentrated toward the edges of the cloud where the effective magnetic field is stronger, an effect which is enhanced as $\nu$ is increased (see Fig. 3). Some additional insight into this effect can be gained by using the concept of diffused vorticity [38], which is applicable if the there are a large number of vortices in the cloud and consequently a dense vortex lattice (much more so than in the examples shown here). In these conditions, the course grained average of the velocity field $\widetilde{\mathbf{v}}$ associated with the lattice will be a smooth function of the radius $r$. A dense regular array of equally spaced vortices would thus mimic solid-body rotation. Approximating the wave function as $\psi=\sqrt{\rho} e^{i \tilde{S}}$, where $\rho$ and $\tilde{S}$ are, respectively, coarse-grained averages of the density and phase such that $\widetilde{\mathbf{v}}=\frac{\hbar}{M} \nabla \tilde{S}$, we obtain an expression for the energy,

$$
E=\int d \mathbf{r} \rho\left[\frac{\hbar^{2}}{2 M} \frac{|\nabla \rho|^{2}}{\rho}+\frac{M}{2} \mathbf{v}^{\prime} \cdot \mathbf{v}^{\prime}+V+\frac{g \rho}{2}-\mu\right],
$$

where $\mathbf{v}^{\prime}=\widetilde{\mathbf{v}}-\frac{\mathbf{A}}{M}$. Minimizing with respect to $\widetilde{\mathbf{v}}$ we find $\widetilde{\mathbf{v}}=\frac{\mathbf{A}}{M}$, independent of the local density $\rho(r)$, and the average vorticity is given by $\boldsymbol{\nabla} \times \widetilde{\mathbf{v}}=\frac{\mathbf{B}}{M}$.

Thus, it is energetically favorable for the induced vorticity to mimic the effective magnetic field, implying that for a field of the form $B \propto r^{\nu-1}(\nu>1)$ there should be a higher concentration of vortices at a larger radius. This is what we indeed see in Fig. 3. Of course, this analysis takes no account of the vortex-vortex interactions (repulsive for vortices of the same "charge"), which become more important as the vortex density is increased and will tend to restore regularity to the lattice.

\section{SUMMARY}

In the examples considered, we find that the nucleation of vortices is crucially dependent on instabilities in the spectrum of elementary excitations, as is known to be the case for condensates subject to external rotation. The form of the magnetic fields considered here dictates that the dynamics are primarily determined by the surface excitations. It is interesting to speculate whether investigation of vortex generation due to more localized effective magnetic fields could provide a connection in the theory with experiments where the condensate is stirred by an optical "spoon" to create vortices [39] as opposed to rotating bucket type configurations [2].

\section{ACKNOWLEDGMENTS}

This work was supported by the United Kingdom EPSRC. S.M.B thanks the Royal Society and the Wolfson Foundation for financial support. D.G. acknowledges financial support from MEC (Spain, Grant No. TEC2006-10009) and Govern Balear (Grant No. PROGECIB-5A).
[1] A. J. Leggett, Quantum Liquids (Oxford University Press, Oxford, 2006).

[2] K. W. Madison, F. Chevy, W. Wohlleben, and J. Dalibard, Phys. Rev. Lett. 84, 806 (2000).

[3] L. Pitaevskii and S. Stringari, Bose-Einstein Condensation (Oxford University Press, Oxford, 2003).

[4] C. J. Pethick and H. Smith, Bose-Einstein Condensatiion in Dilute Gases (Cambridge University Press, Cambridge, England, 2002).

[5] S. Sinha and Y. Castin, Phys. Rev. Lett. 87, 190402 (2001).
[6] T.-L. Ho, Phys. Rev. Lett. 87, 060403 (2001).

[7] N. R. Cooper, N. K. Wilkin, and J. M. F. Gunn, Phys. Rev. Lett. 87, 120405 (2001).

[8] U. R. Fischer and G. Baym, Phys. Rev. Lett. 90, 140402 (2003).

[9] F. Chevy, K. W. Madison, and J. Dalibard, Phys. Rev. Lett. 85, 2223 (2000).

[10] R. Onofrio, C. Raman, J. M. Vogels, J. R. Abo-Shaeer, A. P. Chikkatur, and W. Ketterle, Phys. Rev. Lett. 85, 2228 (2000).

[11] O. M. Maragò, S. A. Hopkins, J. Arlt, E. Hodby, G. Hechen- 
blaikner, and C. J. Foot, Phys. Rev. Lett. 84, 2056 (2000).

[12] M. Olshanii, Phys. Rev. Lett. 81, 938 (1998).

[13] M. D. Girardeau and E. M. Wright, Phys. Rev. Lett. 84, 5691 (2000).

[14] P. Öhberg and L. Santos, Phys. Rev. Lett. 89, 240402 (2002).

[15] V. Bretin, S. Stock, Y. Seurin, and J. Dalibard, Phys. Rev. Lett. 92, 050403 (2004).

[16] V. Schweikhard, I. Coddington, P. Engels, V. P. Mogendorff, and E. A. Cornell, Phys. Rev. Lett. 92, 040404 (2004).

[17] M. A. Baranov, K. Osterloh, and M. Lewenstein, Phys. Rev. Lett. 94, 070404 (2005).

[18] J. Ruostekoski, G. V. Dunne, and J. Javanainen, Phys. Rev. Lett. 88, 180401 (2002).

[19] D. Jaksch and P. Zoller, New J. Phys. 5, 56 (2003).

[20] E. J. Mueller, Phys. Rev. A 70, 041603(R) (2004).

[21] G. Juzeliūnas and P. Öhberg, Phys. Rev. Lett. 93, 033602 (2004).

[22] G. Juzeliūnas, P. Öhberg, J. Ruseckas, and A. Klein, Phys. Rev. A 71, 053614 (2005).

[23] K. J. Günter, M. Cheneau, T. Yefsah, S. P. Rath, and J. Dalibard, Phys. Rev. A 79, 011604(R) (2009).

[24] J. Leach, M. R. Dennis, J. Courtial, and M. Padgett, Nature (London) 432, 165 (2004).

[25] M. V. Berry, Proc. R. Soc. London, Ser. A 392, 45 (1984).

[26] S. Franke-Arnold, J. Leach, M. J. Padgett, V. E. Lembessis, D. Ellinas, A. J. Wright, J. M. Girkin, P. Öhberg, and A. S. Arnold, Opt. Express 15, 8619 (2007).
[27] L. Allen, M. W. Beijersbergen, R. J. C. Spreeuw, and J. P. Woerdman, Phys. Rev. A 45, 8185 (1992).

[28] D. R. Murray, S. M. Barnett, P. Öhberg, and D. Gomila, Phys. Rev. A 76, 053626 (2007).

[29] N. G. Parker and C. S. Adams, Phys. Rev. Lett. 95, 145301 (2005).

[30] N. G. Parker, R. M. W. van Bijnen, and A. M. Martin, Phys. Rev. A 73, 061603(R) (2006).

[31] K. W. Madison, F. Chevy, V. Bretin, and J. Dalibard, Phys. Rev. Lett. 86, 4443 (2001).

[32] A. Recati, F. Zambelli, and S. Stringari, Phys. Rev. Lett. 86, 377 (2001)

[33] E. Hodby, G. Hechenblaikner, S. A. Hopkins, O. M. Maragò, and C. J. Foot, Phys. Rev. Lett. 88, 010405 (2001).

[34] A. D. Jackson, G. M. Kavoulakis, and E. Lundh, Phys. Rev. A 72, 053617 (2005).

[35] E. Lundh, J.-P. Martikainen, and K.-A. Suominen, Phys. Rev. A 67, 063604 (2003)

[36] M. Krämer, L. Pitaevskii, S. Stringari, and F. Zambelli, Laser Phys. 12, 113 (2002).

[37] D. R. Tilley and J. Tilley, Superfluidity and Superconductivity (Institiute of Physics, Bristol, 1990).

[38] R. P. Feynman, Progress in Low Temperature Physics (NorthHolland, Amsterdam, 1955).

[39] C. Raman, J. R. Abo-Shaeer, J. M. Vogels, K. Xu, and W. Ketterle, Phys. Rev. Lett. 87, 210402 (2001). 